\title{
pixOL: pixel-wise point spread function engineering for measuring the 3D orientation and 3D location of dipole-like emitters
}

Tingting $\mathrm{Wu}$, Jin Lu and Matthew Lew

Department of Electrical and Systems Engineering, Washington University in St. Louis, Missouri, USA, saint louis, Missouri, United States

The orientation of a single molecule (SM) is influenced by the intermolecular forces between it and its local environment. Measuring the orientation and location of each SM simultaneously maps interactions between molecules at the nanoscale, which can provide critical insights into a variety of biological processes such as the aggregation of amyloid proteins [1] and the motions of molecular motors [2-4]. Fully understanding molecular movements in living cells requires simultaneous 3D orientation and 3D location measurements. The image of an SM produced by a traditional microscope, called its point spread function, contained relatively little information about $3 \mathrm{D}$ orientations and $3 \mathrm{D}$ positions, leading to poor sensitivity.

To improve imaging sensitivity, PSF engineering has been widely used by inserting a phase mask at the back focal plane of a microscope. Applications that have a low signal-to-noise ratio (SNR) or densely labeled samples pose challenges to existing multi-spot PSFs [5-7]. Furthermore, it is desirable to optimize a PSF for a specific sample of interest to achieve optimal imaging performance.

Here we propose an algorithm, termed pixOL, to simultaneously optimize all pixels of a phase mask across two orthogonally polarized imaging channels for applications with a low SNR (Fig. 1(a)). Unlike optimization using Zernike polynomials [8], pixOL can directly take advantage of super-critical fluorescence arising from imaging SMs near a refractive index interface. Using the resulting phase mask (Fig. 1(a) inset) enables simultaneous measurement of 3D orientation and 3D location of an emitter with small statistical variance. One can easily modify the parameters in pixOL to optimize a phase mask to fit their own microscope and application. Using the pixOL PSF, we measure the orientation of Nile red (NR) molecules transiently attached to two supported lipid bilayers (SLBs): DPPC (di(16:0) PC) and DPPC with cholesterol $[9,10]$. The orientations of NR measured by the pixOL PSF reveal the chemical compositions of the SLBs.

We model the SM as a dipole-like emitter (Fig 2(b)) [11-13]. Each emitter's 3D location is represented by $(x, y, h)$. We use polar angle $\theta$ and azimuthal angle $\Phi$ to describe the mean orientation of the emitter during a camera frame, and we use the solid angle $\Omega$ [sr] to describe the wobble of a molecule during the camera acquisition $(\Omega=0$ represents a fixed dipole emitter and $\Omega=2$ pi means a freely rotating, isotropic emitter). For each set of orientation angles $(\theta, \Phi, \Omega)$, there is a unique timeaveraged orientational second moment $M \in \mathrm{R}^{6 \times 1}$ [14]. The pixOL algorithm optimizes the phase mask $\boldsymbol{P}$ with $\mathrm{N} \times \mathrm{N}$ pixels by simultaneously updating all pixels towards the gradient direction that minimizes the Cramér-Rao (CRB) matrix $\boldsymbol{R}_{M} \in \mathrm{R}^{6 \times 6}$ as

$\boldsymbol{P}_{\mathrm{opt}}=\operatorname{argmin}_{\boldsymbol{P} \in \mathrm{R}}{ }^{\mathrm{N} \times \mathrm{N}} \sum \sqrt{ }\left(\operatorname{det}\left(\boldsymbol{R}_{\boldsymbol{M}}(\theta, \Phi, \Omega, \boldsymbol{P})\right)\right)$,

where $\operatorname{det}(\bullet)$ donates the matrix determinant, and the summation is over orientation space. Thus, pixOL converges upon the phase mask that optimizes the precision of measuring $\boldsymbol{M}$. 
With the pixOL phase mask $\boldsymbol{P}_{\text {opt }}$ (Fig 1(a) inset) inserted at the back focal plane of a microscope, emitters at different 3D orientations (Fig. 2(c)(d), emitters 1-6) and at two axial locations (Fig. 2(c)(d), $h=0 \mathrm{~nm}$ and $h=300 \mathrm{~nm}$ ) are easily resolvable from one another in the image plane. Notably, these images are similar in size to the standard diffraction-limited PSF. Thus, the pixOL PSF is suitable for low SNR applications and densely labeled samples. For fixed emitters $(\Omega=0)$ at a glass-water interface, we calculate the average root-mean-square angular error $\sigma_{k}$ as a combined standard deviation of measuring $\theta$ and $\Phi[15]$ and the average standard deviation $\sigma_{\Omega}$ of $\Omega$ using the CRB (Fig. 1(e)(f)). Compared to other orientation-sensing PSFs, namely CHIDO [16], the polarized PSF defocused at 200nm below the coverslip [17], and the tri-spot PSF [7], the pixOL PSF shows the best $3 \mathrm{D}$ orientation estimation precision. We also calculated the lateral $(\mathrm{x}, \mathrm{y})$ localization precision $\sigma_{r}$ and the axial localization precision $\sigma_{h}$ for an isotropic emitter (Fig. $1(\mathrm{~g})(\mathrm{h})$ ). The pixOL PSF has the best axial localization precision and good lateral precision following CHIDO.

Using the pixOL PSF, we measure the orientations of Nile red (NR) emitters transiently attached to two SLBs: DPPC (di(16:0) PC) and DPPC with 40\% cholesterol (Fig. 2(a)(b)). Within SLBs, the orientation of NR is greatly influenced by its local environment [18]. Added cholesterol (chol) condenses lipid acyl chains and stabilizes lipid membranes [19,20]. We use a sparsity-promoting maximum-likelihood estimator to estimate the brightness, 2D position $(x, y)$, and 3D orientation $(\theta, \Phi, \Omega)$ of individual NR within each frame [21]. We find the orientations of NRs in DPPC with chol are tilted further away from the coverslip plane than NRs in DPPC without chol (Fig. 2(b)). The orientations in DPPC with chol have a narrower distribution indicating a more stabilized lipid membrane environment (Fig. 2(c)).

Here, we demonstrate an algorithm (pixOL) that optimizes a phase mask pixel-by-pixel to efficiently encode the 3D orientation and 3D location of an emitter into the shape of the PSF. The resulting pixOL PSF, optimized for a microscope with two polarized detection channels, shows superior measurement precision for both 3D orientation and 3D position compared to existing methods. In addition, the pixOL PSF is similar in size to the diffraction-limited PSF, enabling it to be used with samples optimized for standard localization microscopy with minimal changes. Our orientation measurements of NR confirm that pixOL can visualize the chemical compositions of lipid membranes [18]. 
(a)

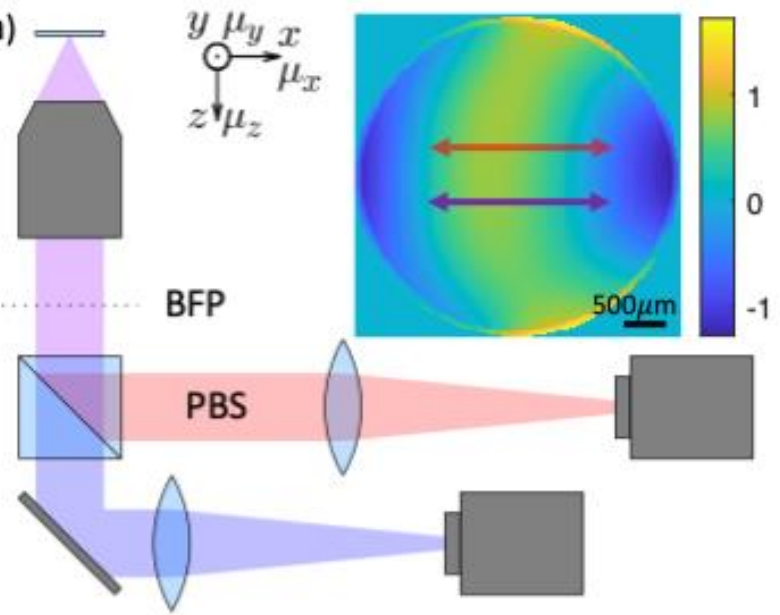

(c)
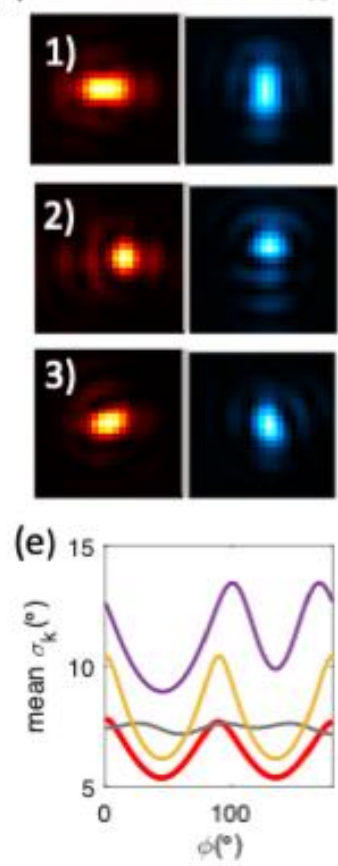

$\mathrm{h}=0 \mathrm{~nm}$
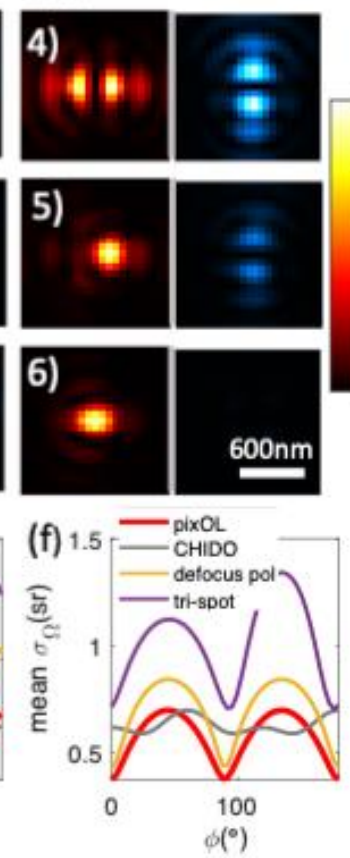

(d)
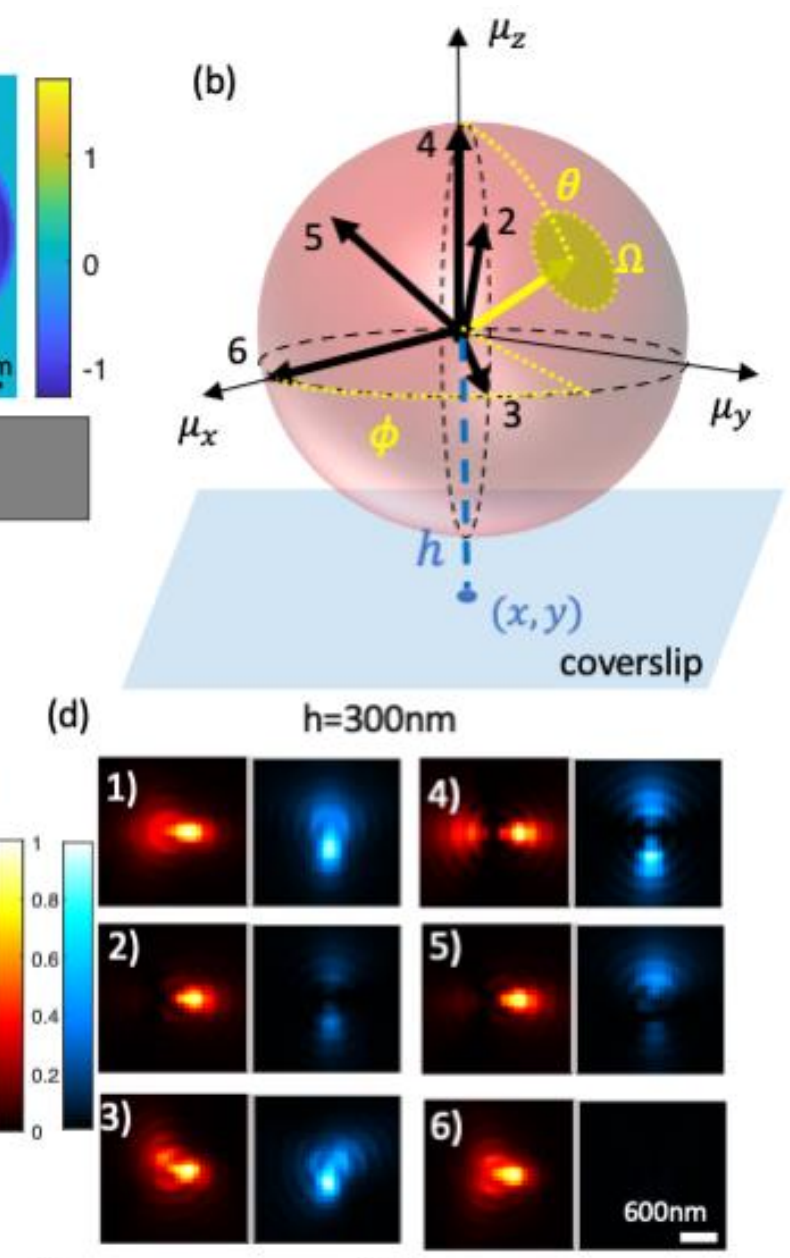

(g)
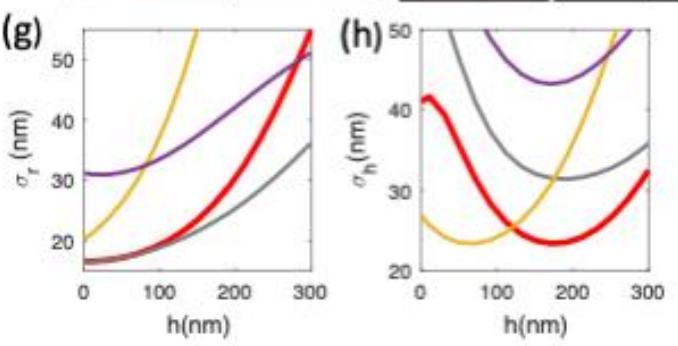

Figure 1. Fig. 1. PSF engineering with pixOL. (a) A dual-channel microscope splits collected fluorescence into two orthogonally polarized channels using a polarizing beam splitter (PBS). The pixOL-optimized phase mask (for emitters near a water-glass interface emitting 380 signal photons and 2 background photons per pixel) is placed at the back focal plane (BFP). Inset: phase mask with polarization directions indicated. Colorbar: phase (rad). (b) The orientation of a transition dipole is parameterized by an azimuthal angle $\Phi \in[-$ pi,pi), a polar angle $\theta \in[0$, pi/2] and a rotational diffusion-associated wobbling area $\Omega \in[0,2 \mathrm{pi}]$. The 3D location of an emitter is parameterized by lateral location $(\mathrm{x}, \mathrm{y})$ and axial location h. (c-d) pixOL PSFs for six emitters whose orientations are shown in (b), located (c) at the coverslip and (d) $300 \mathrm{~nm}$ above the coverslip. Pseudo-colored red and blue images correspond to the two polarization channels shown in (a). The intensities of each red-blue image pair are normalized. Emitter 1: an isotropic emitter with $\Omega=2$ pi; emitter

2: $\theta=30^{\circ}, \Phi=45^{\circ}, \Omega=0$; emitter 3: $\theta=90^{\circ}, \Phi=45^{\circ}, \Omega=0$; emitter 4: $\theta=0^{\circ}, \Omega=0$; emitter 5: $\theta=30^{\circ}, \Phi=0^{\circ}, \Omega=0$; emitter 6: $\theta=90^{\circ}, \Phi=0^{\circ}, \Omega=0$; (e,f) Best-possible precision of measuring $3 \mathrm{D}$ angles (e) $\sigma \mathrm{k}$ and (f) $\sigma \Omega$ averaged over uniformly 
sampled $\theta$ for fixed emitters. (g,h) Best possible localization precisions $(\mathrm{g})$ or and $(\mathrm{h})$ oh calculated for isotropic emitters. Red: pixOL; grey: CHIDO; yellow: polarized PSF defocused at 200nm below the coverslip (defocus pol); purple: tri-spot. Root-mean square angular error $\sigma \mathrm{k}$ is a combined standard

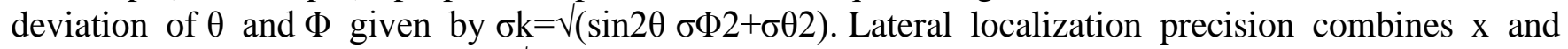
y localization precision using $\sigma \mathrm{r}=\sqrt{ }(\sigma \times 2+\sigma \mathrm{y} 2)$.

(a) DPPC
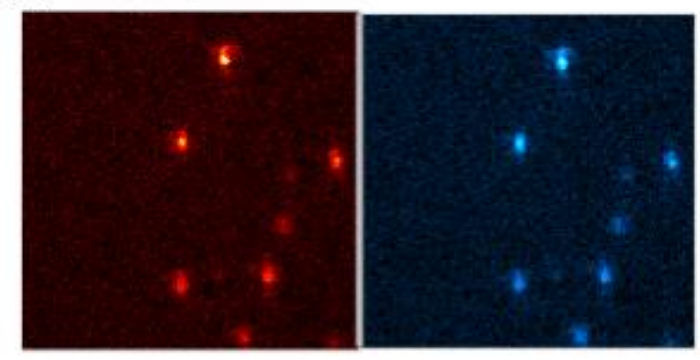

(b) DPPC $+40 \% \mathrm{chol}$

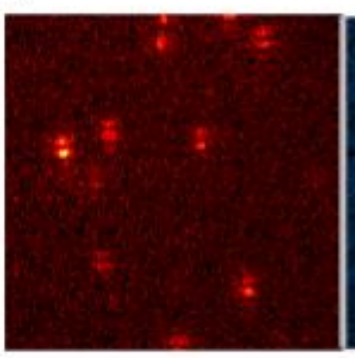

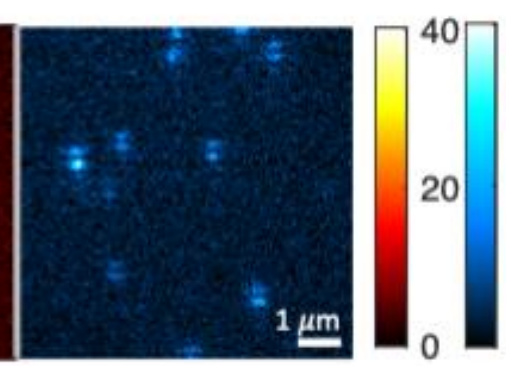

(c)

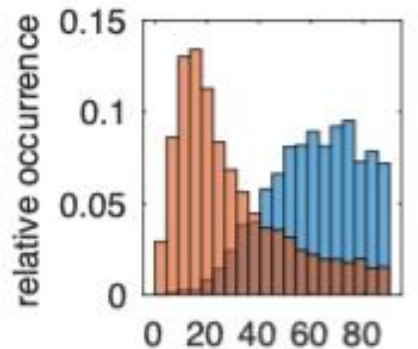

$\theta\left(^{\circ}\right)$

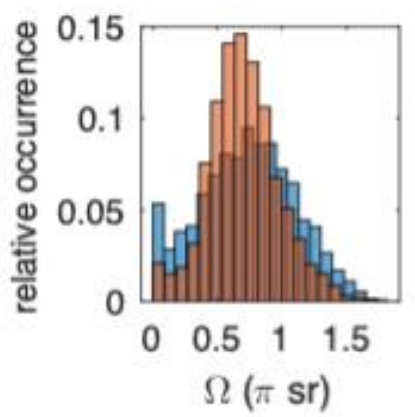

(d)
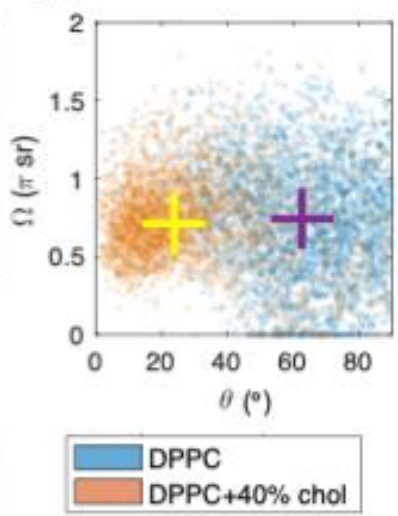

Figure 2. Fig 2. 3D orientation measurements of Nile red (NR) within supported lipid bilayers (SLBs). (a,b) Raw images of NRs within (a) DPPC and (b) DPPC with 40\% chol SLBs. Colorbar: photons. Scale bar: $1 \mu \mathrm{m}$. (c) Histograms of measured 3D orientations (polar angle $\theta$, wobbling solid angle $\Omega$ ) of NRs. Blue: DPPC; orange: DPPC with $40 \%$ chol. (d) Scatter plot of the measured $\theta$ and $\Omega$ of NRs in DPPC (blue) and DPPC with chol (orange). Purple cross: median orientations of NRs in DPPC; yellow cross: median orientations in DPPC with $40 \%$ chol.

\section{References}

1. [1] T. Ding, T. Wu, H. Mazidi, O. Zhang, and M. Lew, Optica 7, 602-607 (2020).

2. [2] H. Sosa, E. J. G. Peterman, W. E. Moerner, and L. S. B. Goldstein, Nat. Struct. Biol. 8, 540544 (2001).

3. [3] J. N. Forkey, M. E. Quinlan, M. Alexander Shaw, J. E. T. Corrie, and Y. E. Goldman, Nature 422, 399-404 (2003).

4. [4] J. F. Beausang, D. Y. Shroder, P. C. Nelson, and Y. E. Goldman, Biophys. J. 104, 1263-1273 (2013).

5. [5] A. S. Backer, M. P. Backlund, A. R. von Diezmann, S. J. Sahl, and W. E. Moerner, Appl. Phys. Lett. 104, 193701 (2014).

6. [6] A. S. Backer, M. P. Backlund, M. D. Lew, and W. E. Moerner, Opt. Lett. 38, 1521 (2013).

7. [7] O. Zhang, J. Lu, T. Ding, and M. D. Lew, Appl. Phys. Lett. 113, 031103 (2018).

8. [8] Y. Shechtman, S. J. Sahl, A. S. Backer, and W. E. Moerner, ” Phys. Rev. Lett. 113, 1-5 (2014).

9. [9] A. Sharonov and R. M. Hochstrasser, " Proc. Natl. Acad. Sci. USA 103, 18911-18916 (2006).

10. [10] C. Kuo and R. M. Hochstrasser, J. Am. Chem. Soc. 133, 4664-4667 (2011). 
11. [11] L. Novotny and B. Hecht, Principles of Nano-Optics (Cambridge University, 2006).

12. [12] T. Chandler, H. Shroff, R. Oldenbourg, and P. la Rivière, JOSA A 36, 1334-1345 (2018).

13. [13] T. Chandler, H. Shroff, R. Oldenbourg, and P. la Rivière, JOSA A 36, 1346-1360 (2019).

14. [14] A. S. Backer and W. E. Moerner, J. Phys. Chem. B 118, 8313-8329 (2014).

16. [15] A. Nehorai and E. Paldi, IEEE Transactions on signal processing 42, (1994).

17. [16] V. Curcio, L. A. Alemán-Castañeda, T. G. Brown, S. Brasselet, and M. A. Alonso, Nat. Commun. 11, 1-13 (2020).

18. [17] O. Zhang and M. D. Lew, JOSA A (2020).

[18] J. Lu, H. Mazidi, T. Ding, O. Zhang, and M. Lew, Angew. Chem. Int. Ed. (2020).

[19] T. Róg and I. Vattulainen, Chem. Phys. Lipids 184, 82-104 (2014).

[20] E. Sezgin, I. Levental, and C. Eggeling, Nat. Rev. Mol. Cell Biol. 18, 361 (2017).

[21] H. Mazidi, E. S. King, O. Zhang, A. Nehorai, and M. D. Lew, International Symposium on Biomedical Imaging, 325-329 (2019). 